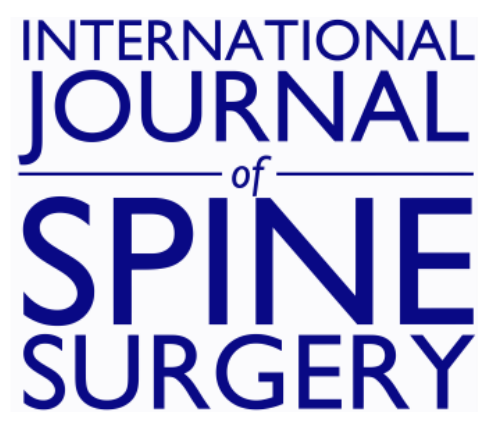

\title{
Stress Reduction in Adjacent Level Discs via Dynamic Instrumentation: A Finite Element Analysis
}

Antonio E. Castellvi, Hao Huang, Tov Vestgaarden, Sunil Saigal, Deborah H. Clabeaux and David Pienkowski

Int J Spine Surg 2007, 1 (2) 74-81

doi: https://doi.org/10.1016/SASJ-2007-0004-RR

http://ijssurgery.com/content/1/2/74

This information is current as of April 25, 2023.

Email Alerts Receive free email-alerts when new articles cite this article. Sign up at:

http://ijssurgery.com/alerts

The International Journal of Spine Surgery

2397 Waterbury Circle, Suite 1,

Aurora, IL 60504, Phone: +1-630-375-1432 


\title{
Stress Reduction in Adjacent Level Discs via Dynamic Instrumentation: A Finite Element Analysis
}

\author{
Antonio E. Castellvi, MD, Hao Huang, PhD, Tov Vestgaarden, PhD, \\ Sunil Saigal, PhD, Deborah H. Clabeaux, RN, and David Pienkowski, PhD
}

\begin{abstract}
Background

Conventional (rigid) fusion instrumentation is believed to accelerate the degeneration of adjacent discs by increasing stresses caused by motion discontinuity. Fusion instrumentation that employs reduced rod stiffness and increased axial motion, or dynamic instrumentation, may partially alleviate this problem, but the effects of this instrumentation on the stresses in the adjacent disc are unknown. We used a finiteelement model to calculate and compare the stresses in the adjacent-level disc that are induced by rigid and dynamic posterior lumbar fusion instrumentation.

Methods

A 3-dimensional finite-element model of the lumbar spine was obtained that simulated flexion and extension. The L5-S1 segment of this model was fused, and the L4-L5 segment was fixed with rigid or dynamic instrumentation. The mechanical properties of the dynamic instrumentation were determined by laboratory testing and then used in the finite-element model. Peak stresses in the lumbar discs were calculated and compared.
\end{abstract}

Results

The reduced-stiffness component of the dynamic instrumentation was associated with a $1 \%$ to $2 \%$ reduction in peak compressive stresses in the adjacent-level disc (at $45^{\circ}$ flexion), and the increased axial motion component of this instrumentation reduced peak disc stress by $8 \%$ to $9 \%$. Areas of disc tissue exposed to $80 \%$ of peak stresses of $6.17 \mathrm{MPa}$ were $47 \%$ less for discs adjacent to dynamic instrumentation than for those adjacent to rigid instrumentation.

\section{Conclusions}

Reduced stiffness and increased axial motion of dynamic posterior lumbar fusion instrumentation designs result in an approximately $10 \%$ cumulative stress reduction for each flexion cycle. The effect of this stress reduction over many cycles may be substantial.

\section{Clinical Relevance}

The cumulative effect of this reduced amplitude and distribution of peak stresses in the adjacent disc may partially alleviate the problem of adjacent-level disc degeneration.

Key Words disc degeneration, spine fusion, posterior instrumentation, disc stresses, adjacent disc. $\boldsymbol{S A S}$ Journal. Spring 2007;1:74-81. DOI: SASJ-2007-0004-RR

\section{INTRODUCTION}

Fusion of adjacent vertebrae is widely used for treating degenerative disc disease, but this procedure does not always alleviate pain ${ }^{1}$ and has a degree of comorbidity. ${ }^{2}$ Use of rigid posterior instrumentation commonly accompanies fusion to prevent motion and aid fusion healing. However, such rigid fixation is believed to accelerate the radiographically-observed degeneration of the discs adjacent to the fused segments, because of the increased stresses caused by the abrupt stiffness and motion discontinuity. ${ }^{3-7}$ As an alternative to rigid fixation, different methods of "soft" or "dynamic", 10 stabilization have emerged. ${ }^{11}$ Regardless of the name used, these stabilization methods feature some type of less-than-rigid instrumentation design connected to modified pedicle screws for the purpose of gaining more favorable movement and load transmission across nonfused segments. Less-than-rigid instrumentation seeks to distribute motion rather than eliminate it and thereby to reduce the likelihood of adjacent-level disc disease while improving the long-term outcome of lumbar fusion procedures. ${ }^{12}$

The efficacy of dynamic stabilization remains controversial and is therefore a suitable topic for continuing investigation. ${ }^{13-16}$ Although several clinical-outcome studies describe preliminary results obtained from the use of dynamic stabilization, ${ }^{1,8,17-19}$ these studies lack a randomized controlled design, a statistically adequate sample size, or long-term follow-up data that would enable the clinical efficacy of these methods to be properly evaluated. ${ }^{10}$ Early data suggests that the results are at least no worse than those observed from rigid instrumentation. ${ }^{1}$ Information is also lacking from a scientific perspective because dynamic stabilization 
methods have largely been developed from clinical suggestions instead of engineering design efforts, and thus the biomechanics of these methods remain relatively unquantified.

The purpose of the present study was to (1) quantify the biomechanics of a conventional rigid (hereafter "rigid") and 1 specific type of dynamic instrumentation when biomechanically tested in a simulated laboratory model, (2) use these data in a finite-element model of a fused and fixed lumbar spine to calculate the flexion-induced peak stresses in the adjacentlevel discs, and (3) compare these results to determine if a biomechanical basis exists for believing that the reduced stiffness and increased axial motion conferred by dynamic instrumentation can alter the stresses in adjacent-level discs.

\section{MATERIALS AND METHODS}

\section{Study Design}

This laboratory study used both standardized compressive testing of dynamic instrumentation on an established lumbar spinal segment model $^{20}$ and a finite-element modeling technique, which enabled quantification of the stresses induced in an established model of lumbar spinal discs ${ }^{21}$ as a function of instrumentation design (rigid vs dynamic). This experimental design - stiffness testing followed by finite-element analysesis consistent with previous studies. ${ }^{22,23}$

\section{Biomechanical Testing}

Both rigid and dynamic (Isobar TTL; Scient'X USA Inc, Maitland,Florida)posteriorlumbarspinalfusion instrumentation were mechanically tested in a polyethylene model of the lumbar spine. This model was developed from the work of Cunningham et al. ${ }^{20}$ and was modified according to American Society for Testing and Materials protocol F1717. This testing quantified the stiffness of the mechanical damper used in the subsequent modeling efforts. Each type of instrumentation was applied across 2 simulated lumbar segments (L4-L5-S1): the L5-S1 segment was the intended segment requiring rigid fixation in both cases, but the difference in instrumentation occurred at the L4-L5 level. One group of specimens (control) had rigid instrumentation across the L4-L5 level; the other group of specimens (experimental) had dynamic instrumentation across the L4-L5 level. Six specimens were prepared and tested for each of the 2 groups (12 tests total).

Quasi-static axial compressive testing to the approximate end of the elastic limit was performed at an actuator movement rate of $1 \mathrm{~cm} / \mathrm{min}$ on an Instron 1331 materials testing system (Instron Corp, Canton, Mass). Actuator displacement and axial load data were acquired with an Instron System 8800 data capture system, and these measurements were used to calculate the elastic stiffness values of all constructs. Axial motion (when present) was also acquired electronically and quantified from the load-displacement curves. The data obtained were then used in the finite-element model.

Finite-Element Modeling

A three-dimensional (3D) finite-element model of the lumbar spine (L1-L5, including discs) was developed by first obtaining a validated finite-element mesh $^{21}$ for the L3-L5 spine section (Figure 1). The geometry was developed from a series of computed tomography scans of the L4 vertebra of a 44-year-old male with no pathologies. ${ }^{21}$ The L4 mesh was then replicated to model the other lumbar spine vertebrae. This model of L1-L5, previously developed and validated by Smit et al., ${ }^{21}$ consists of a series of 5 dimensionally equivalent L4 vertebrae. This resulting mesh of L1-L5 vertebrae was positioned such that the angle between the inferior surface of L2 and the superior surface of L5 was $40^{\circ}$. This model consisted of a fused (totally rigid) L5-S1 segment and an L4-L5 segment that was modeled to imitate fixation with either rigid or dynamic instrumentation. The dimensions for the instrumentation used in this model were obtained from direct measurement of exemplar instrumentation (Isobar TTL). The fused segment L5-S1 was modeled by specifying the material properties of the L5-S1 disc to be the same as those of cortical bone. Adjacent pairs of vertebrae were connected by intervertebral discs that were modeled as a nucleus in the center surrounded by 3 to 4 rings of annulus fibrosus. The nucleus typically occupies approximately $30 \%$ to $50 \%$ of the area of the disc. Therefore, the fraction used for the nucleus in the model obtained was $43 \%{ }^{24}$ (Figure 2). The entire finite-element model contained 18,128 three-dimensional 8-node linear brick elements.

Loading of the model was accomplished by combined flexion or extension plus axial loading. The axial load of $400 \mathrm{~N}$ was applied as a "follower" load, thereby allowing the axial load to follow the motion of the spine. The model simulated forward flexion at discrete angular increments of $15^{\circ}, 30^{\circ}$, and $45^{\circ}$ and a backward extension of $15^{\circ}$ by applying relative angular displacements between the L1-L2, L2-L3, and L3-L4 segments, respectively, on the basis of values equal to those obtained from a normal spine during forward flexion and backward extension. ${ }^{25}$

The damper of the dynamic instrumentation, located between the instrumented L5 and L4 vertebrae, permitted the upper segment of the fixation rod to have reduced stiffness and limited axial micromotion. These 2 features of this damper mechanism were modeled by employing a softer segment (having variable stiffness values, all of which were less than those of titanium alloy) placed in series with an axial motion connector (which allowed axial motion only; Figure 3). Two parameters, R and $\mathrm{G}$, were used in this model to quantify the reduced stiffness and the axial micromotion of the damper mechanism, respectively. The damper is an integral component of the TTL device, which is responsible for these 2 features.

\section{The parameter}

$$
\mathrm{R}=\mathrm{K}_{\text {rigid }} / \mathrm{K}_{\text {dynamic }}
$$

was used to quantify the reduced stiffness of the damper. This dimensionless stiffness ratio quantified how much stiffer the rigid instrumentation was than the dynamic instrumentation. 
Figure 1

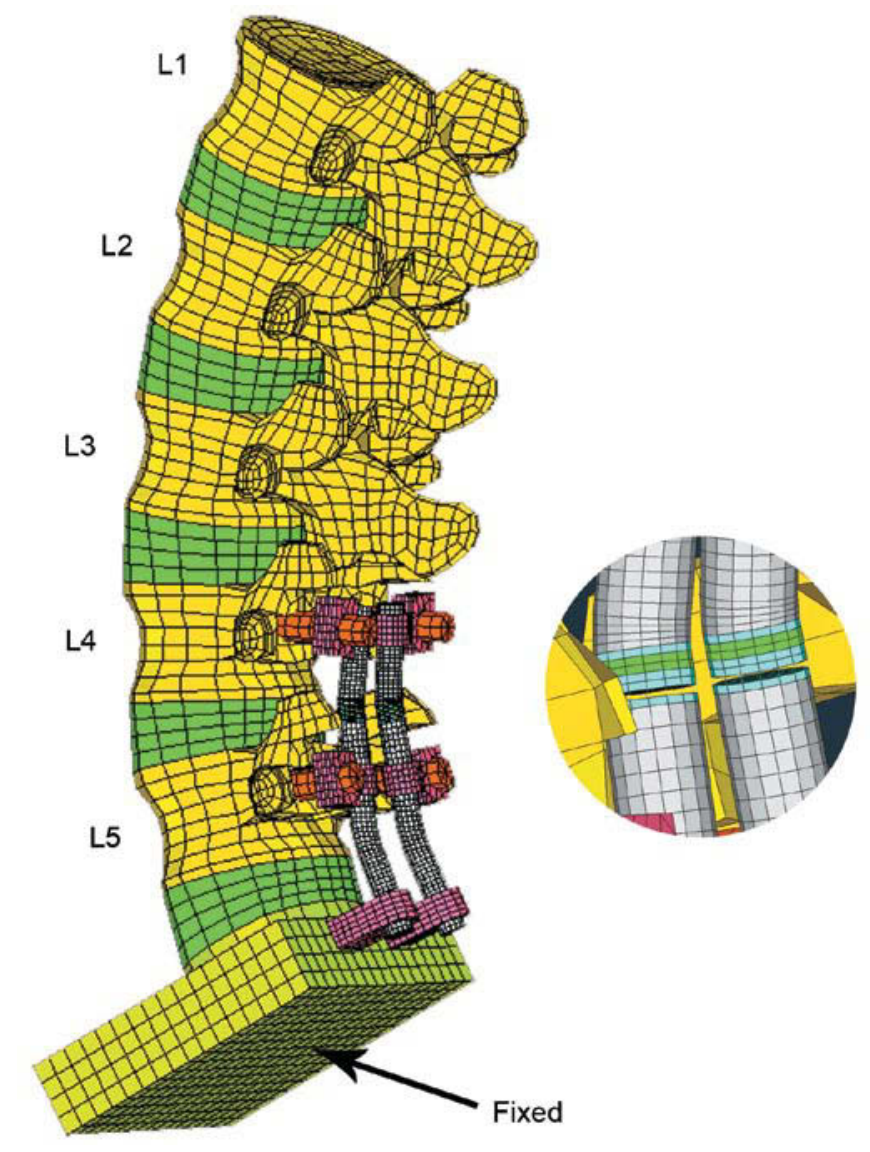

Note. Isometric view of the finite-element mesh adapted from a model created and validated by Smit et al. ${ }^{20}$ from which our model of the lumbar spine was derived and to which the semirigid instrumentation was applied.

Isometric view of the finite element mesh of lumbar spine and semirigid rods.

\section{Figure 2}

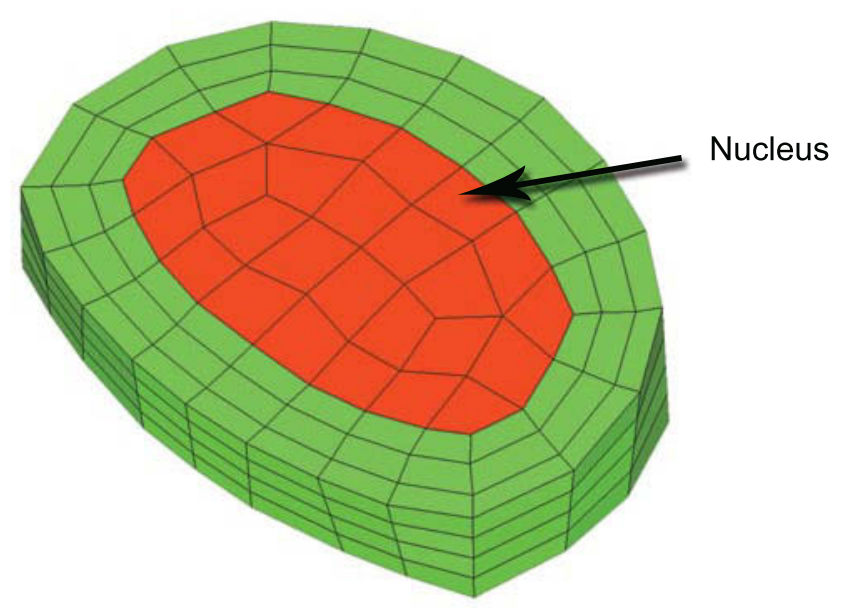

Note. Model shows the annulus fibrosis (green color; outer 3 layers of mesh elements) and the nucleus pulposis (red color; inner mesh elements).

Isometric view of an intervertebral disc.
Figure 3

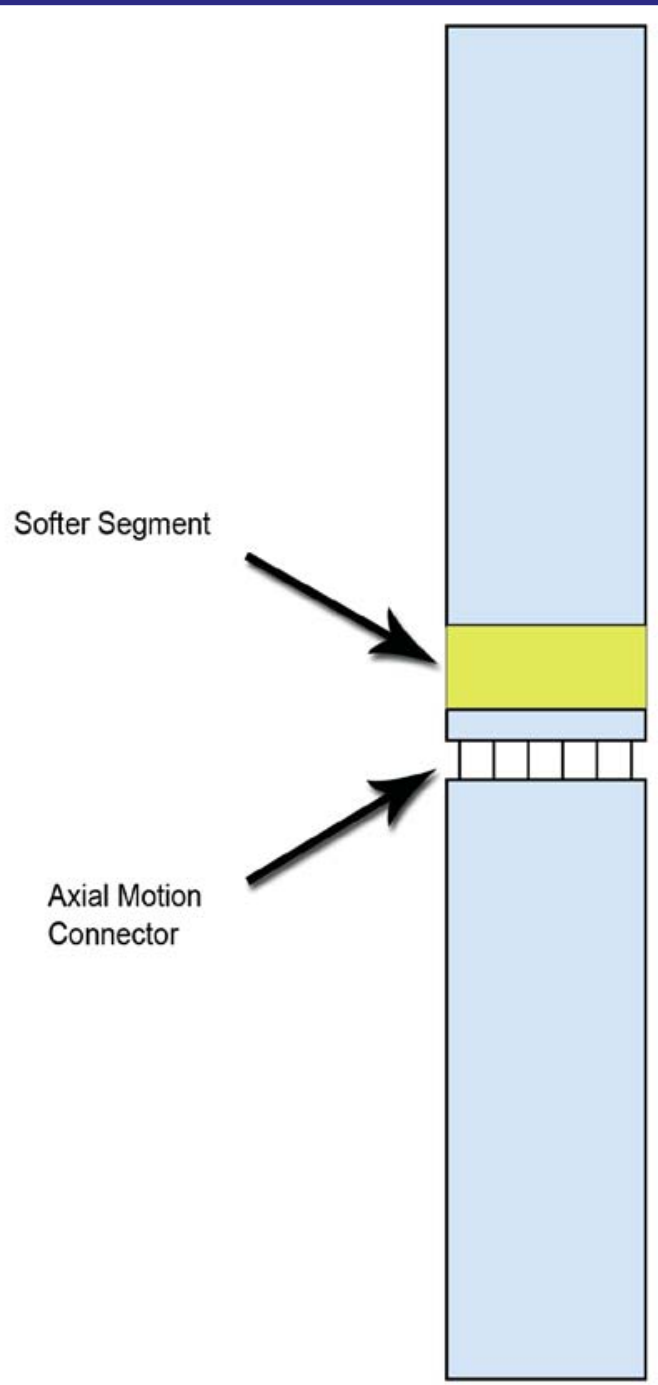

Note. Expanded schematic illustration of the mechanical components of the damper element of the dynamic instrumentation component shown in Figure 1. Objects above and below the denoted elements are rigid rods.

The damper model of the dynamic instrumentation.

The $\mathrm{K}_{\text {rigid }}$ term of equation 1 represents the stiffness of the rigid instrumentation, and the $\mathrm{K}_{\text {dynamic }}$ term represents the stiffness of the dynamic instrumentation. Values for $\mathrm{K}_{\text {rigid }}$ and $\mathrm{K}_{\text {dynamic }}$ were obtained from the material properties of titanium alloy and the variable reduced stiffness of the softer segment. The $G$ parameter was defined as the maximum axial motion allowed by the damper mechanism. To study the effects of axial motion on the resulting pressures induced within the disc, 5 discrete maximum-allowable axial displacements $(0-0.8 \mathrm{~mm}$ in $0.2-\mathrm{mm}$ increments) were used in the model. Changes to both $\mathrm{R}$ and $\mathrm{G}$ permitted the changes in pressure within the disc to be quantified as a result of varying instrumentation elastic stiffness and axial micromotion. Before reaching the maximum axial motion, the damper also functioned as an axial spring with a stiffness of $175 \mathrm{KN} / \mathrm{m}$ (calculated from the product manual accompanying the Isobar TTL instrumentation). 
The inferior portion of the sacrum was modeled as a block, and the lower surface of the block was considered fixed. A static compressive (follower) load of $400 \mathrm{~N}$ was axially applied to the superior surface of the L1 vertebra, and this load was maintained perpendicular to the superior surface of the L1 segment throughout axial-load-induced deformation. All components in the assembly shown (Figure 1) were modeled with linear elastic materials. The material properties assigned to these components ${ }^{21,25}$ in the finiteelement model are shown in Table 1. Peak stress values in the disc, as well as the areas of the $2 \mathrm{D}$ projections of the $3 \mathrm{D}$ volumes of disc tissue exposed to $>80 \%$ of peak stress volumes, were calculated for varying values of $R$ and $G$ with commercially available finite-element analysis software (ABAQUS/Standard; ABAQUS Inc, Pawtucket, RI).

\section{Table 1}

Material Properties Obtained From Sources Listed and Used in the Finite Element Model

\begin{tabular}{lcc}
\hline \multicolumn{1}{c}{ Material } & Young's Modulus, GPa & Poisson's Ratio \\
\hline Cortical Bone & 12 & 0.3 \\
Cancellous Bone & 3 & 0.2 \\
Fibrous & 0.03 & 0.45 \\
Nucleus & 0.001 & 0.49 \\
Steel & 190 & 0.3 \\
Titanium & 116 & 0.33 \\
\hline
\end{tabular}

Note. $\mathrm{GPa}=$ gigaPascals. Poisson's ratio is dimensionless.

\section{STATISTICAL METHODS}

Analysis of the mechanical test data was performed with StatView (SAS Institute, Cary, NC). Data were checked for normality with the Komolgorov-Smirnov test, and then the mean values were compared with Student's $t$ test. $P$ values $<.05$ were considered to be indicative of significant differences.

\section{RESULTS}

Mean value $( \pm$ SD) of the elastic stiffness (axial load divided by actuator displacement) of the rigid instrumentation was $21,960 \pm 8,034 \mathrm{~N} / \mathrm{mm}$; the mean elastic stiffness of the dynamic instrumentation was less than one third this value $(P=.01)$, or $6,169 \pm 1,298 \mathrm{~N} / \mathrm{mm}$. These data yielded $\mathrm{R}$ and $\mathrm{G}$ values for the rigid instrumentation of 1 (control stiffness value) and 0 (meaning no axial micromotion [obtained from the manufacturer]), respectively, whereas the $R$ and $G$ values for the dynamic instrumentation were 3.6 and $0.4 \mathrm{~mm}$, respectively. Other values for $\mathrm{R}$ and $\mathrm{G}$ were also used in the model calculations to compute the effect of alternative values for elastic stiffness and axial micromotion (Tables 2 and 3).

Calculated values are shown for the peak von Mises stresses induced within the L3-L4 disc for the 400-N axial load applied with each of the 2 instrumentation designs at each of the 4 flexion/extension positions $\left(15^{\circ}, 30^{\circ}\right.$, and $45^{\circ}$ flexion and $15^{\circ}$ extension) and for varying values of $\mathrm{R}$ and $\mathrm{G}$ (Table 2 ). The data
Table 2

\begin{tabular}{|c|c|c|c|c|c|}
\hline \multicolumn{6}{|c|}{ Peak Calculated Stress (MPa) in the L3-L4 Disc } \\
\hline \multirow[b]{2}{*}{$\mathrm{R}$ (Ratio) } & \multicolumn{5}{|c|}{$\mathrm{G}, \mathrm{mm}$} \\
\hline & 0.0 & 0.2 & 0.4 & 0.6 & 0.8 \\
\hline \multicolumn{6}{|c|}{$45^{\circ}$ angle } \\
\hline 1 & 7.7096 & 7.5364 & 7.3715 & 7.2067 & 7.0422 \\
\hline 3.6 & 7.6376 & 7.4578 & 7.2866 & 7.1157 & 6.9453 \\
\hline 10 & 7.5644 & 7.3867 & 7.2174 & 7.0485 & 6.8800 \\
\hline 44 & 7.3416 & & & & \\
\hline \multicolumn{6}{|c|}{$30^{\circ}$ angle } \\
\hline 1 & 5.0483 & 4.8767 & 4.7133 & 4.5503 & 4.3882 \\
\hline 3.6 & 4.9999 & 4.8211 & 4.6511 & 4.4814 & 4.3123 \\
\hline 10 & 4.9524 & 4.7754 & 4.6069 & 4.4388 & 4.2712 \\
\hline 44 & 4.8044 & & & & \\
\hline \multicolumn{6}{|c|}{$15^{\circ}$ angle } \\
\hline 1 & 2.4776 & 2.3078 & 2.1472 & 2.0859 & 2.0859 \\
\hline 3.6 & 2.4532 & 2.2759 & 2.1077 & 1.9404 & 1.9101 \\
\hline 10 & 2.4303 & 2.2542 & 2.0870 & 1.9209 & 1.8515 \\
\hline 44 & 2.3569 & & & & \\
\hline \multicolumn{6}{|c|}{$-15^{\circ}$ angle } \\
\hline 1 & 4.2420 & 4.0428 & 3.8508 & 3.8508 & 3.8508 \\
\hline 3.6 & 4.2348 & 4.0251 & 3.8066 & 3.7431 & 3.7431 \\
\hline 10 & 4.2215 & 4.0126 & 3.7947 & 3.7093 & 3.7093 \\
\hline 44 & 4.2085 & & & & \\
\hline
\end{tabular}

Note. $\mathrm{MPa}=$ megaPascals. Table entries are the peak stresses $(\mathrm{MPa})$ induced in the $\mathrm{L} 3-\mathrm{L} 4$ disc superior to the dynamic instrumentation component as calculated from the finite element model as a function of (1) angle (+ = flexion, $-=$ extension), (2) dimensionless stiffness ratio $R$, and ( 3 ) axial motion parameter $G$.

showed that the use of dynamic instrumentation was associated with a $5.5 \%$ reduction in peak stress within the L3-L4 disc and a $16.7 \%$ increase in peak stress within the L4-L5 disc (Table 3) compared with the rigid instrumentation at $45^{\circ}$ of flexion. It was also observed that maintaining the $\mathrm{G}$ value at 0.0 (allowing no axial micromotion) but allowing the stiffness of the proximal segment of the dynamic instrumentation to decrease caused a reduction in the peak stress in the L3-L4 disc of approximately $1 \%$ to $2 \%$. Alternatively, maintaining the same stiffness of this proximal segment as that found in the rigid case (maintaining the $\mathrm{R}$ value at 1 but increasing the axial micromotion, i.e., increasing the $\mathrm{G}$ value) resulted in reducing the peak stress in the L3-L4 disc by approximately $8 \%$ to $9 \%$. Thus, increasing the $\mathrm{G}$ parameter (specifically, increasing axial micromotion) was more effective in reducing the peak stress in the L3-L4 disc than was decreasing the $\mathrm{R}$ parameter (specifically, decreasing the rod stiffness). These effects were also observed at $15^{\circ}$ and $30^{\circ}$ of flexion as well as at $15^{\circ}$ of extension, but less prominently (Figure 4). The minimal value for peak stress in the L3-L4 disc in the $45^{\circ}$ flexion case was achieved for $\mathrm{R}$ and $\mathrm{G}$ values of 10 and $0.8 \mathrm{~mm}$, respectively. 
Table 3

\begin{tabular}{|c|c|c|c|c|c|}
\hline \multicolumn{6}{|c|}{ Peak Calculated Stress (MPa) in the L4-L5 Disc } \\
\hline \multirow[b]{2}{*}{$\mathrm{R}$ (Ratio) } & \multicolumn{5}{|c|}{$\mathrm{G}, \mathrm{mm}$} \\
\hline & 0.0 & 0.2 & 0.4 & 0.6 & 0.8 \\
\hline \multicolumn{6}{|c|}{$45^{\circ}$ angle } \\
\hline 1 & 2.5972 & 2.7377 & 2.8713 & 3.0043 & 3.1369 \\
\hline 3.6 & 2.7141 & 2.8765 & 3.0317 & 3.1872 & 3.3429 \\
\hline 10 & 2.7601 & 2.9242 & 3.0812 & 3.2386 & 3.3964 \\
\hline 44 & 2.9633 & & & & \\
\hline \multicolumn{6}{|c|}{$30^{\circ}$ angle } \\
\hline 1 & 1.7221 & 1.8579 & 1.9873 & 2.1165 & 2.2448 \\
\hline 3.6 & 1.8010 & 1.9591 & 2.1105 & 2.2624 & 2.4147 \\
\hline 10 & 1.8309 & 1.9912 & 2.1448 & 2.2990 & 2.4537 \\
\hline 44 & 1.9626 & & & & \\
\hline \multicolumn{6}{|c|}{$15^{\circ}$ angle } \\
\hline 1 & 0.8522 & 0.9844 & 1.1106 & 1.1588 & 1.1588 \\
\hline 3.6 & 0.8921 & 1.0470 & 1.1955 & 1.3443 & 1.3713 \\
\hline 10 & 0.9067 & 1.0642 & 1.2153 & 1.3667 & 1.4300 \\
\hline 44 & 0.9717 & & & & \\
\hline \multicolumn{6}{|c|}{$-15^{\circ}$ angle } \\
\hline 1 & 0.4319 & 0.8208 & 1.2029 & 1.2029 & 1.2029 \\
\hline 3.6 & 0.4803 & 0.8214 & 1.1828 & 1.2882 & 1.2882 \\
\hline 10 & 0.5368 & 0.8616 & 1.2055 & 1.3410 & 1.3410 \\
\hline 44 & 0.8796 & & & & \\
\hline
\end{tabular}

Note. $\mathrm{MPa}=$ megaPascals. Table entries are the peak stresses $(\mathrm{MPa})$ induced in the L3-L4 disc superior to the dynamic instrumentation component as calculated from the finite element model as a function of (1) angle (+ = flexion, $-=$ extension), (2) dimensionless stiffness ratio $R$, and (3) axial motion parameter $G$.

To graphically visualize the stress reduction caused by reduced stiffness and increased axial micromotion associated with dynamic instrumentation, we contrasted the stress levels in the L3-L4 disc located above rigid instrumentation with those of the same disc located above dynamic instrumentation that have the optimal dynamic parameters $(\mathrm{R}=10, \mathrm{G}=0.8 \mathrm{~mm}$; Figure 5). The volume of L3-L4 disc tissue located above the dynamic instrumentation that was exposed to stresses of 6.17 $\mathrm{MPa}$ or greater was $47 \%$ less than the volume of L3-L4 disc tissue located above the rigid instrumentation that was exposed to stresses of $6.17 \mathrm{MPa}$ or greater. The stress value $6.17 \mathrm{MPa}$ was $80 \%$ of the peak stress in the L3-L4 disc located above the rigid instrumentation when calculated at $45^{\circ}$ of flexion.

\section{DISCUSSION}

Reduced stiffness and increased axial motion of dynamic posterior lumbar spinal fixation instrumentation resulted in both lower peak stresses and smaller volumes of tissue exposed to high-amplitude stresses in simulated adjacent-level discs. Although the stress reduction effect was small (approximately $10 \%$ cumulatively for a single forward flexion), this is important because this benefit will be repeated over many loading cycles
Figure 4

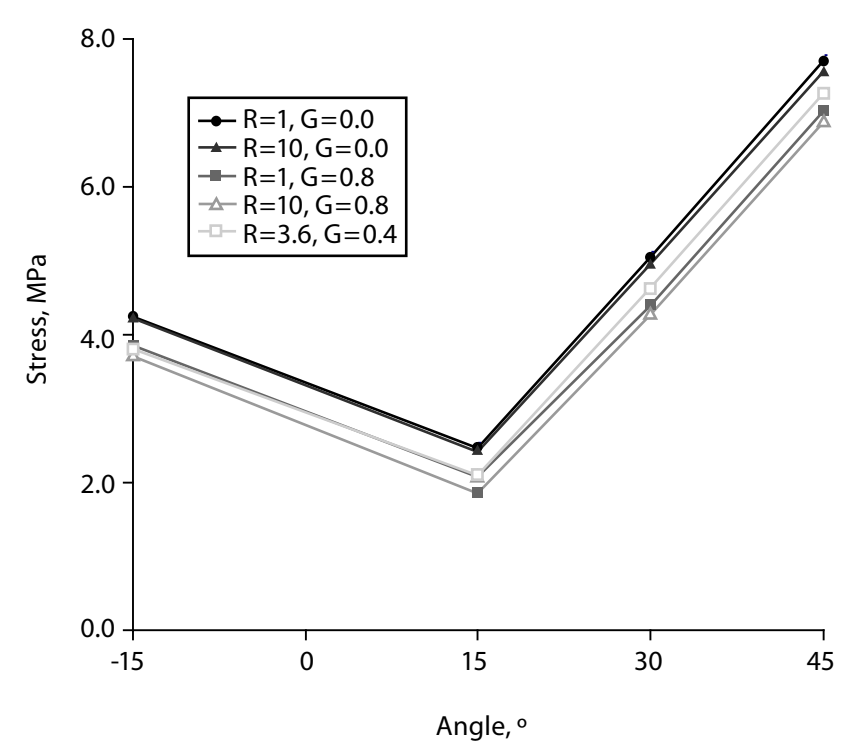

Note. Representative values for the calculated stresses induced in the L3-L4 disc as function of 1 of 4 different flexion/extension angles (abscissa) and for varying indicated color-coded values of relative stiffness (R-parameter values) and axial motion (G-parameter values).

Comparison of stress in L3-L4 with different variables for $\mathrm{R}$ and $\mathrm{G}$.

Figure 5
$R=1 / 10, G=0.8 \mathrm{~mm}(\operatorname{Max} 6.88 \mathrm{MPa})$

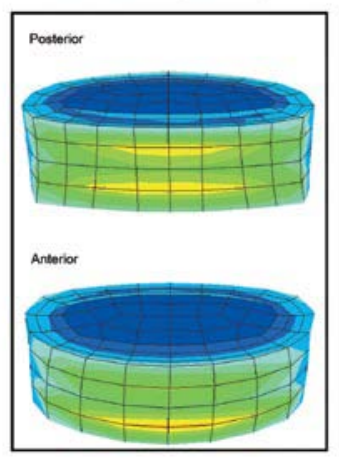

Rigid Rod (Max 7.71 MPa)

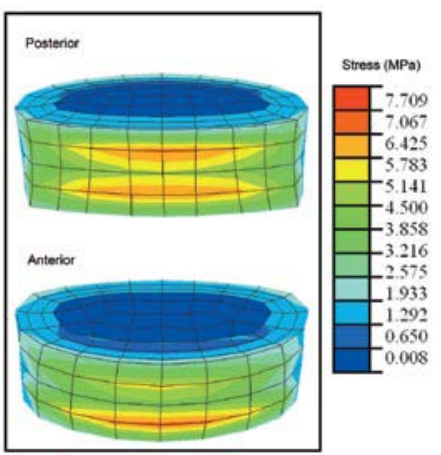

Note. Anterior-posterior views of calculated stress distribution in the L3-L4 disc at a $45^{\circ}$ flexion angle for discs associated with rigid instrumentation (right side) and dynamic instrumentation (left side). Dynamic instrumentation has $1 / 10$ stiffness of the rigid instrumentation, i.e., $R=0.1$ and $0.8 \mathrm{~mm}$ axial motion. Peak induced stress in the disc associated with the rigid instrumentation is $7.71 \mathrm{MPa}$; peak induced stress in the disc associated with the dynamic instrumentation is $6.88 \mathrm{MPa}$.

Stress distribution of $\mathrm{L} 3-\mathrm{L} 4$ at $45^{\circ}$ flexion.

(1-10 million/year). Classic material-fatigue studies show that small reductions in peak load amplitude produce substantial increases in material longevity, and this finding is substantiated by analogous studies conducted in cadaveric lumbar vertebrae. ${ }^{26}$ Although the reduced stiffness and increased axial motion also increased the peak stress in the L4-L5 disc by up to $28 \%$, this load increase should be considered in light of the peak stress 
amplitude in the L4-L5 disc, which was 2 to 3 times less than that in the adjacent L3-L4 disc. The reduced stiffness and increased axial motion of dynamic instrumentation also allows some rotation of the $\mathrm{L} 4$ vertebra with respect to $\mathrm{L} 5$. This rotation is not permitted by rigid instrumentation designs. To achieve the same overall level of flexion when both types of devices are used, the L3-L4 disc experiences smaller rotation demands when this type (Isobar TTL) of dynamic instrumentation is used. Reduced rotation then leads to a corresponding stress reduction in this disc.

Only a few published studies are reasonably comparable to the present study. Three of these used cadaveric spinal segments that were mechanically tested in vitro in conjunction with another type of dynamic instrumentation (Dynesis; Zimmer Spine, Minneapolis, Minnesota). All showed that this type of dynamic instrumentation can favorably alter load transmission and movement yet can also provide adequate stability. None of these studies quantified the within-disc pressure changes that remain at the heart of adjacent segment degeneration. ${ }^{27-29}$ Another study used computational models to compare materials selection but not device design. That study also focused on overall mechanical stability and load transmission rather than on pressures within the disc. ${ }^{23} \mathrm{~A}$ fifth study used a finite-element method to compute pressures within adjacent discs but did not study the effects of dynamic instrumentation. ${ }^{30}$ The study that was most similar to the present study also used a finiteelement model of the lumbar discs but concluded that dynamic instrumentation does not alter pressures within the discs. ${ }^{31}$ This disparity in findings may reflect the mechanical performance differences between the Isobar system (present study) and the Dynesis system (Zander et al. ${ }^{31}$ ).

It is important to note that dynamic instrumentation also permits axial distraction, which in turn changes the center of rotation. In the case of the 2 instrumented segments A and B (Figure 6a), if axial distraction (i.e., increase of the interpedicular distance) is permitted, then the center of rotation shifts and falls within the L4-L5 disc and not on the posterior side of the posterior lateral ligament (Figure 6a). When no axial motion is allowed, the center of rotation is located at the level of the damper (which is acting as a type of hinge; Figure 6b). This shift in the center of rotation reduces the effective moment arm for $\mathrm{L} 4$ rotation, which in turn causes a reduced moment and lower stresses in the L3-L4 disc, because L1 will have the same displacement in both cases. This allows a more physiological motion than can otherwise be obtained with instrumentation that does not allow distraction.

Decreasing the $\mathrm{R}$ parameter alone has the effect of reducing the stiffness of the material resisting the rotation, whereas decreasing the $\mathrm{G}$ parameter alone has the effect of adjusting the axis of rotation for L4. Our numerical results demonstrate that within the range of values for stiffness and axial motion (parameters $\mathrm{R}$ and $\mathrm{G}$ ) that we used, moving the center of rotation anteriorly is more effective in reducing stress amplitudes in the adjacent-level disc than is reducing the elastic stiffness of the instrumentation. Although the particular type of dynamic
Figure 6
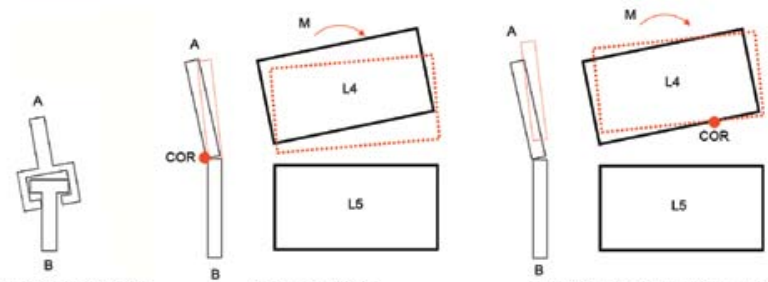

Note. Sagittal view of a schematic illustration of the damper mechanism that shows 2 approaches regarding how rotation can be obtained for instrumentation that allows (a) dynamic motion, (b) pure bending only with no axial motion (note the location of the center of rotation), or (c) bending with axial compression/extension (note the altered [more physiological] location of the center of rotation).

Two approaches to generating $2^{\circ}$ of rotation: (a) dynamic instrumentation, (b) allowing bending, and (c) allowing extension/compression.

instrumentation studied has both features - anterior translation of the center of rotation and reduced elastic stiffness - the former feature is considered to be clinically more important.

Increased load demands at the adjacent-level disc accompanying fusion have been associated with accelerated degeneration of that disc in animal models ${ }^{32}$ and are also associated with adjacent-level disc problems in humans. ${ }^{6}$ Rigid fixation has been associated with increased pressures within the disc that are as much as $73 \%$ greater in adjacent cervical discs. ${ }^{33}$ Other researchers suggest that not just the amplitude, but also the altered pattern of loading may have a role in this process of adjacent-level disc disease. ${ }^{11}$ Given the current findings, some argue that adequate proof of the difference between rigid and dynamic stabilization has not yet been established ${ }^{4}$; others claim that the lack of differences provides support for the concept. ${ }^{10}$ Answers may come from adequately powered, longitudinal, randomized, controlled clinical trials of dynamic versus conventional instrumentation. An important point is that "dynamic" is an appellation for a generic class of load-sharing fixation instrumentation; differences in the design and materials of such devices result in varying levels of stiffness and motion. Outcomes of computational or in vivo studies employing dynamic devices are likely to be different owing to their biomechanical heterogeneity. Only the resulting clinical studies will enable those with superior performance to be identified.

Limitations of the present study include the less-than-ideal anatomical model used. The lumbar vertebrae employed in this finite-element model were not size-adjusted for the various vertebral levels, but were all identical and derived from the dimensions of an L4 vertebral body. However, this model was developed and validated previously ${ }^{21}$ and thus is not considered a major limitation because the focus of the study was the comparative, not absolute, differences in pressures within the disc. Also, because loading deforms the in vivo spine, the load likely does not remain perpendicular. However, for the model used in this study, it was assumed to remain perpendicular. This assumption introduces a limitation to the absolute accuracy of 
the internal stress results reported, but the magnitude of this error is considered small and the comparative (between rigid and dynamic stabilization instrumentation) effects are believed to be negligible. The model used also did not include the effects of degenerative disc material properties, strain-dependent disc swelling pressures/material permeability, or nonlinear elastic material behavior. Although these may be important from an absolute perspective to understand the behavior of individual discs, ${ }^{34}$ their relative contribution in the present study involving comparison of 2 different fixation types is considered insignificant.

Assuming that adjacent-level disc deterioration is partially caused by repetitive high-amplitude loading and nonphysiologic axes of rotation, reduced elastic bending stiffness and increased axial motion attributable to an anteriorly shifted axis of rotation in posterior instrumentation will more favorably distribute the motion demands of the lumbar spine. This finding supports emerging clinical evidence that such mechanical alterations to posterior spinal fixation devices have a beneficial effect on disc tissue and thereby delay the onset, reduce the severity of, or prevent entirely the phenomenon of accelerated adjacent-level disc deterioration. Therefore, reducing the stiffness, increasing the axial motion, and anteriorly translating the axis of rotation of posterior spinal fixation instrumentation may be part of the solution to the problem of adjacent-level disc degeneration.

Antonio E. Castellvi, MD, Hao Huang, PhD, Tov Vestgaarden, PhD, Sunil Saigal, PhD, Deborah H. Clabeaux, RN, and David Pienkowski, PhD

From the Florida Orthopaedic Institute, Tampa (Castellvi and Clabeaux); the Exxonmobil Upstream Research Company, Houston, Texas (Huang); the Department of Civil and Environmental Engineering, University of South Florida, Tampa (Vestgaarden and Saigal); and the Center for Biomedical Engineering and the Department of Orthopaedic Surgery, University of Kentucky, Lexington (Pienkowski).

Address correspondence and reprint requests to Deborah H. Clabeaux, RN, Florida Orthopaedic Institute, 2727 Martin Luther King Blvd, Tampa, FL 33607 (email: dclabeaux@ floridaortho.com).

Antonio Castellvi is a consultant to Scient'X, Maitland, Florida.

We acknowledge research support and equipment from Scient'X USA and the support of both the Department of Civil and Environmental Engineering and the Research Oriented Computing Center of the University of South Florida. We also acknowledge research support from the Center for Biomedical Engineering and the Department of Orthopaedic Surgery at the University of Kentucky.

This submission was received March 7, 2007, and accepted for publication June 1, 2007.

Institutional review board approval was not required for this study.

\section{REFERENCES}

1. Schnake KJ, Schaeren S, Jeanneret B. Dynamic stabilization in addition to decompression for lumbar spinal stenosis with degenerative spondylolisthesis. Spine. 2006;31:442-449.

2. Phillips FM, Voronov LI, Gaitanis IN, et al. Biomechanics of posterior dynamic stabilizing device (DIAM) after facetectomy and discectomy. Spine J. 2006;6:714-722.
3. Kumar MN, Jacquot F, Hall H. Long-term follow-up of functional outcomes and radiographic changes at adjacent levels following lumbar spine fusion for degenerative disc disease. Eur Spine J. 2001;10:309313.

4. Korovessis P, Papazisis Z, Koureas G, et al. Rigid, semirigid versus dynamic instrumentation for degenerative lumbar spinal stenosis: a correlative radiological and clinical analysis of short-term results. Spine. 2004;29:735-742.

5. International Society of Biomechanics Web site. Available at: http:// www.isbweb.org. Accessed July 20, 2004.

6. Gillet P. The fate of the adjacent motion segments after lumbar fusion. $J$ Spinal Disord Tech. 2003;16:338-345.

7. Park P, Garton HJ, Gala VC, et al. Adjacent segment disease after lumbar or lumbosacral fusion: review of the literature. Spine. 2004;29:19381944.

8. Brechbuhler D, Markwalder TM, Braun M. Surgical results after soft system stabilization of the lumbar spine in degenerative disc diseaselong-term results. Acta Neurochir (Wien). 1998;140:521-525.

9. Wild A, Jaeger M, Bushe C, et al. Biomechanical analysis of Graf's dynamic spine stabilisation system ex vivo. Biomed Tech (Berl). 2001;46:290-294.

10. Stoll TM, Dubois G, Schwarzenbach O. The dynamic neutralization system for the spine: a multi-center study of a novel non-fusion system. Eur Spine J. 2002;11 Suppl 2:S170-178.

11. Mulholland RC, Sengupta DK. Rationale, principles and experimental evaluation of the concept of soft stabilization. Eur Spine J. 2002;11 Suppl 2:S198-205.

12. Sengupta DK. Dynamic stabilization devices in the treatment of low back pain. Orthop Clin North Am. 2004;35:43-56.

13. Grob D, Benini A, Junge A, et al. Clinical experience with the Dynesys semirigid fixation system for the lumbar spine: surgical and patientoriented outcome in 50 cases after an average of 2 years. Spine. 2005;30:324-331.

14. Cakir B, Ulmar B, Koepp H, et al. Posterior dynamic stabilization as an alternative to dorsoventral fusion in spinal stenosis with degenerative instability [in German]. Z Orthop Ihre Grenzgeb. 2003;141:418-424.

15. Markwalder TM, Wenger M. Dynamic stabilization of lumbar motion segments by use of Graf's ligaments: results with an average follow-up of 7.4 years in 39 highly selected, consecutive patients. Acta Neurochir (Wien). 2003;145:209-214; discussion 14.

16. Saxler G, Wedemeyer C, von Knoch M, et al. Follow-up study after dynamic and static stabilization of the lumbar spine [in German]. $Z$ Orthop Ihre Grenzgeb. 2005;143:92-99.

17. Bose B. Anterior cervical arthrodesis using DOC dynamic stabilization implant for improvement in sagittal angulation and controlled settling. $J$ Neurosurg Spine. 2003;98:8-13.

18. Putzier M, Schneider SV, Funk J, et al. Application of a dynamic pedicle screw system (Dynesys) for lumbar segmental degenerationscomparison of clinical and radiological results for different indications [in German]. Z Orthop Ihre Grenzgeb. 2004;142:166-173.

19. Kanayama M, Hashimoto T, Shigenobu K, et al. Adjacent-segment morbidity after Graf ligamentoplasty compared with posterolateral lumbar fusion. J Neurosurg Spine. 2001;95:5-10.

20. Cunningham BW, Sefter JC, Shoo Y, et al. Static and cyclical 
biomechanical analysis of pedicle screw spinal constructs. Spine. 1993;18:1677-1688.

21. Smit TH, Odgaard A, Schneider E. Structure and function of vertebral trabecular bone. Spine. 1997;22:2823-2833.

22. Martinez JB, Oloyede VO, Broom ND. Biomechanics of load-bearing of the intervertebral disc: an experimental and finite element model. Med Eng Phys. 1997;19:145-156.

23. Vena P, Franzoso G, Gastaldi D, et al. A finite element model of the L4-L5 spinal motion segment: biomechanical compatibility of an interspinous device. Comput Methods Biomech Biomed Engin. $2005 ; 8: 7-16$

24. White AA, Panjabi M. Clinical Biomechanics of the Spine. 2nd ed. Philadelphia: Lippincott; 1990.

25. Goel VK, Monroe BT, Gilbertson LG, et al. Interlaminar shear stresses and laminae separation in a disc. Finite element analysis of the L3-L4 motion segment subjected to axial compressive loads. Spine. 1995;20:689-698.

26. Adams MA, Freeman BJ, Morrison HP, et al. Mechanical initiation of intervertebral disc degeneration. Spine. 2000;25:1625-1636.

27. Schmoelz W, Huber JF, Nydegger T, et al. Dynamic stabilization of the lumbar spine and its effects on adjacent segments: an in vitro experiment. J Spinal Disord Tech. 2003;16:418-423.

28. Niosi CA, Zhu QA, Wilson DC, et al. Biomechanical characterization of the three-dimensional kinematic behaviour of the Dynesys dynamic stabilization system: an in vitro study. Eur Spine J. 2006;15:913-922.

29. Xu HZ, Wang XY, Chi YL, et al. Biomechanical evaluation of a dynamic pedicle screw fixation device. Clin Biomech (Bristol, Avon). 2006;21:330-336.

30. Chen CS, Cheng CK, Liu CL, et al. Stress analysis of the disc adjacent to interbody fusion in lumbar spine. Med Eng Phys. 2001;23:483-491.

31. Zander T, Rohlmann A, Burra NK, et al. Effect of a posterior dynamic implant adjacent to a rigid spinal fixator. Clin Biomech (Bristol, Avon). 2006;21:767-774.

32. Kroeber M, Unglaub F, Guegring T, et al. Effects of controlled dynamic disc distraction on degenerated intervertebral discs: an in vivo study on the rabbit lumbar spine model. Spine. 2005;30:181-187.

33. Eck JC, Humphreys SC, Lim TH, Jeong ST, Kim JG, Hodges SD, An HS. Biomechanical study on the effect of cervical spine fusion on adjacent-level intradiscal pressure and segmental motion. Spine. 2002;27(22):2431-2434.

34. Natarajan RN, Williams JR, Andersson GB. Recent advances in analytical modeling of lumbar disc degeneration. Spine. 2004;29:2733-2741. 